\title{
Dynamic Analysis of the Walking Process of Parallel Foot - Wheeled Robot based on Kane Method
}

\author{
ZHAO Jiangbo ${ }^{1,}$ a , WANG Shuanglei ${ }^{2, b}$ \\ ${ }^{1}$ Beijing Institute of Technology, Beijing 100081, China \\ ${ }^{2}$ Beijing Institute of Technology, Beijing 100081, China \\ a zhaojiangboo@bit.edu.cn, ${ }^{\mathrm{b}}$ 17801056162@163.com
}

\begin{abstract}
Keywords: Parallel robot, Dynamics, Kane method.
Abstract. A research has been carried out on the dynamic analysis and simulation of parallel robots due to the situation where parallel robots tend to break down on account of large stress during movements. In order to study the force condition of the robot on the move, a mathematical model, based on the Kane method, has been built and the driving force of each electric cylinder has been calculated by computer programming. In the meantime, a dynamic simulation model and a simulation control system are established on the basis of Adams and Matlab and through analyzing the joint simulation, we can obtain the driving force of each cylinder. The gap between the simulation results and the results obtained by the mathematical model is within $7 \%$, which verifies the correctness of the Kane dynamic system and gives provide the foundation for future studies of dynamics and structure optimization.
\end{abstract}

\section{Introduction}

In 1965, Stewart platform mechanism was put forward by American scholar Stewart for the first time and it is used as a flight simulator. Stewart platform was applied in spacecraft docking and wheel assembly robot, and virtual axis machine ${ }^{[1]}$ and optical detection ${ }^{[2]}$, radar field ${ }^{[3]}$ and so on through the continuous development in recent years.

Four Stewart platform mechanisms were used as the four legs of the parallel foot-wheel quadruped robot which is described in this paper, And at the bottom of Stewart platform is a wheel 。 Such a structure provides two modes of operation: wheeled robot mode for controlling wheel speed. And a quadruped robot model that controls the movement of four Stewart platform mechanisms. The Stress situation of wheeled robot is relatively simple, in this paper, the dynamics of quadruped robots is studied.

The structure of robot is complex and the cost of the robot is high. How to maintain the life of the robot is an important research field in the robot field. Therefore, it is of great value and significance to study the stress of each structure of the robot to avoid its paralysis caused by excessive force in the process of motion.

Dynamical model is the foundation of parallel robot to realize the dynamic simulation, dynamic analysis, dynamic optimization design and control of parallel robot, which plays an important role in the research. The Lagrangian method, Newton-Euler method, Gauss method, Kane method, spin number (even number method) and Roberson Wittenberg method ${ }^{[4-6]}$ are used to establish the dynamic model.

M Ahmadi ${ }^{[7]}$ used the first kind of Lagrangian equation to establish the dynamics model of the parallel robot; Chen Xiulong et al. ${ }^{[8]}$ used the Newton-Euler method to establish the kinetic equation of the parallel mechanism, and used to study the rigid body dynamics modeling of the 4-UPS-UPU 5-DOF spatial parallel mechanism.G Cheng, X Shan ${ }^{[9]}$ used the kane method to analyze the kinematics of the 4-DOF parallel hip joint simulator; On the basis of Kane's equation, Liu Minjie et $\mathrm{al}^{[10]}$. proposed the sub-structure model of the parallel robot dynamics.

Compared with other methods, the Kane method can avoid the calculation of the kinetic functions and their derivatives in the process of deriving the kinetic equation, and can be transformed into the inner product and the outer product of the vector. Besides, it can use computer-aided computing, 
which greatly simplifying the calculation of the workload. Therefore, the Kane method is used to analyze the kinematics of the parallel foot-wheeled quadruped robot.

Stress Analysis Based on Kane Method. The establishment of Kane equation is based on the concepts of generalized velocity, partial velocity, deflection velocity, generalized active force and generalized inertia force proposed by Professor Kane. The basic principle of the method is to select the appropriate generalized velocity, determine the partial velocity and the angular velocity, and then calculate the generalized dynamic force and the generalized inertial force, and then give the Kane dynamic equation according to the Dahl-Lagrangian principle ${ }^{[11]}$.

Kinematic analysis. Parallel foot-wheeled robot and its structural diagram are shown in Figure 1, 2 respectively. The four axes of the robot are numbered 1,2, 3 and 4 in clockwise order. The coordinate system is shown in Fig. 1, where $\mathrm{O}$ is the center point of the upper platform and the base coordinate system $\mathrm{O}-\mathrm{XYZ}$ is fixed to the upper platform. $\mathrm{O}_{1}, \mathrm{O}_{2}, \mathrm{O}_{3}, \mathrm{O}_{4}$ are located on the platform, respectively, four center of Stewart platform . $\mathrm{O}_{1}, \mathrm{O}_{2}, \mathrm{O}_{3}, \mathrm{O}_{4}$ corresponding to the center of lower platform, the respective coordinate system were connected to the corresponding platform .In this paper, the robot will be a step-by-step crawl gait to move, four legs of the same law of motion, so take 1 legs for analysis.

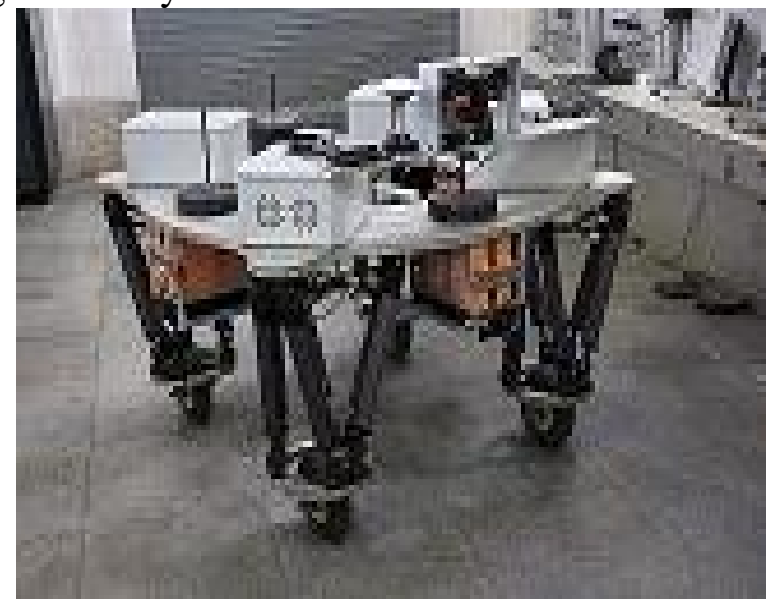

Fig.1 parallel Legged robot

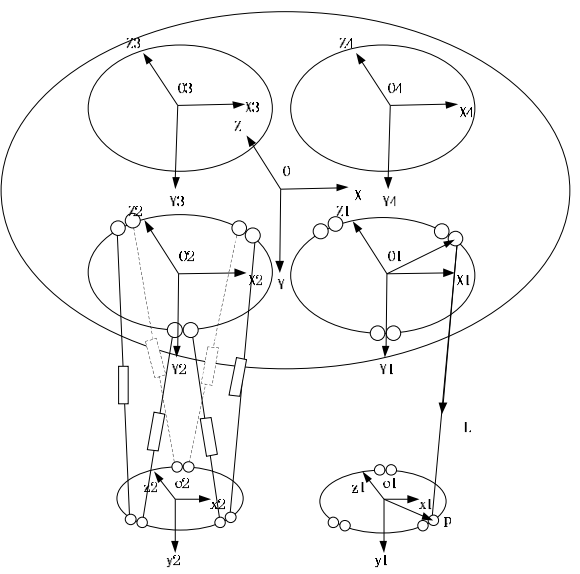

Fig.2 Structure diagram of parallel Legged robot

For the lower platform and the leverage of an intersection $\mathrm{p}$, in the base coordinate system, said:

$$
p=R r_{o_{1}}+r_{c} \text {. }
$$

Where,$R$ is a direction transformed cosine matrix represented by an angle of $R-P-Y ; r_{01}$ is the position vector of $\mathrm{p}$ point in the lower platform coordinate system $\mathrm{o} 1-\mathrm{x} 1 \mathrm{y} 1 \mathrm{z} 1 ; \mathrm{r}_{\mathrm{c}}$ is the position vector of the center point of the lower platform in the base coordinate system. Assuming $r 1=\mathrm{Rr}_{\mathrm{o} 1}$, then

$$
p=r_{1}+r_{c} \text {. }
$$

We can get the speed of $\mathrm{p}$ point

$$
\delta=\delta+\delta_{c}=\omega \times r_{1}+\phi_{c} \text {. }
$$

Where $\omega$ is the angular velocity of rotation of the lower platform.

For the p-point velocity there are relations as follows

$$
\delta=\&+\omega_{L} \times s \text {. }
$$

Where $\delta_{\text {is }}$ the velocity component along the L-direction; s is a unit vector in the L-direction, $s=L / l, 1$ is the length of the vector; $\omega_{L}$ is the angular velocity of the rod rotation. Therefore:

$$
\omega_{L}=s \times \delta / l=s \times\left(\omega \times r_{1}+\underset{c}{\phi}\right) / l .
$$


the result of the derivation of the equation (4) is:

$$
\begin{aligned}
& =+\omega_{L} \times\left(\omega_{L} \times p\right)+2 \omega_{L} \times \alpha_{L}+\alpha_{L} \times p . \\
& \alpha_{L}=s \times 2 \omega_{L} \& / l . \\
& L \omega_{L} \cdot \omega_{L} .
\end{aligned}
$$

For the rod L, in fact, the electric cylinder, the lower part of the lower platform connected with the cylinder rod, The upper part of the connection with the upper platform for the cylinder, in this paper will be simplified as a mobile pair, The velocity and acceleration of the centroid of the cylinder are:

$$
\begin{aligned}
& v_{t}=\omega_{L} \times r_{t}=s \times\left(\omega \times r_{1}+\underset{c}{\delta}\right) \times r_{t} / l . \\
& a_{t}=\alpha_{L} \times r_{t}+\omega_{L} \times\left(\omega_{L} \times r_{t}\right) .
\end{aligned}
$$

Where, $r_{t}$ is the vector of the centroid of the cylinder in the base coordinate system.

The velocity and acceleration of the cylinder center of mass are:

$$
\begin{aligned}
& v_{g}=\$ \omega_{L} \times r_{g}=s \cdot\left(\omega \times r_{1}+\underset{c}{\delta}\right) s+s \times\left(\omega \times r_{1}+\alpha_{c}\right) \times r_{g} / l . \\
& a_{g}=\alpha_{L} \times r_{g}+\omega_{L} \times\left(\omega_{L} \times r_{g}\right)+2 \delta \omega_{L} \times s .
\end{aligned}
$$

Where, $r_{g}$ is the vector of the lever centroid in the base coordinate system.

Determination of Deflection Speed and Deflection Speed. Since the system is a complete system, so the generalized rate $\phi_{j}$ is independent of each other, take the generalized rate $\oint_{J}=\left[\begin{array}{llllll}v_{x} & v_{y} & v_{z} & \omega_{x} & \omega_{y} & \omega_{z}\end{array}\right]^{T} \quad(j=1,2, \cdots \cdots 6)$, Since the electric cylinder is simplified as a moving pair in this paper, it can be obtained that the centroid of the cylinder is the same as the angular velocity of the mass center of the cylinder and the angular velocity of the generalized velocity is:

$$
\omega_{L}^{r}=\left[\omega_{L v}^{r} \omega_{L \omega}^{r}\right]=\left[\frac{s \times E_{3}}{l} \quad \frac{s \times\left(E_{3} \times r_{1}\right)}{l}\right] .
$$

Where $E_{3}$ is the third-order unit vector.

The eccentricity of the centroid of the cylinder for the generalized rate is:

$v_{t}^{r}=\left[v_{t v}^{r} v_{t \omega}^{r}\right]=\omega_{L}^{r} \times r_{t}$.

The eccentricity of cylinder center of mass is:

$v_{g}^{r}=\left[\begin{array}{lll}v_{g v}{ }^{r} & v_{g \omega}{ }^{r}\end{array}\right] \mathcal{E}_{S}+\omega^{r}{ }_{L} \times r_{g}$.

Where, $E^{\&}=\left[s \cdot E_{3} s \cdot\left(E_{3} \times r_{1}\right)\right]$

The velocity and angular velocity of the center of mass about the generalized velocity are:

$$
\begin{aligned}
& v^{r}=\left[\begin{array}{ll}
v_{v}^{r} & v_{\omega}{ }^{r}
\end{array}\right]=\left[\begin{array}{ll}
O_{3} & O_{3}
\end{array}\right] . \\
& \omega^{r}=\left[\begin{array}{ll}
\omega_{v}{ }^{r} & \omega_{\omega}{ }^{r}
\end{array}\right]=\left[\begin{array}{ll}
O_{3} & E_{3}
\end{array}\right] .
\end{aligned}
$$

Where, $\mathrm{O}_{3}$ is the third-order matrix.

Establish dynamic equation. Suppose the generalized dominant force for the generalized rate $\oiint_{J}=\left[\begin{array}{llllll}v_{x} & v_{y} & v_{z} & \omega_{x} & \omega_{y} & \omega_{z}\end{array}\right]^{T} \quad(j=1,2, \cdots \cdots 6)$ is $F_{j}=\left[\begin{array}{ll}F_{v} & F_{\omega}\end{array}\right]$, generalized inertial force is 
$F_{j}^{*}=\left[F_{v}^{*} F_{\omega}^{*}\right]$, where, the generalized dominant force for the generalized rate $\oint_{j}\left[\begin{array}{lll}v_{x} & v_{y} & v_{z}\end{array}\right]^{T}$ is $F_{v}$, the generalized inertial force is $F_{v}^{*}$.

$$
\begin{aligned}
& F_{v}-\sum_{i=1}^{6}\left(m_{g} g \cdot v_{i=1}{ }^{\gamma}+m_{z} g \cdot v_{m p}{ }^{\prime}\right)+M g \cdot v_{v}^{\prime}+R F_{v} \cdot F_{i j}+\sum_{i=1}^{6} F_{i} \cdot v_{v}^{\prime} .
\end{aligned}
$$

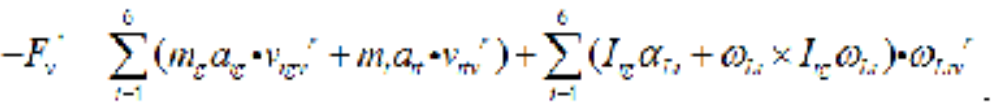

$$
\begin{aligned}
& +\left(I_{t r} \alpha_{t, r}+\omega_{7,} \times I_{i}, \omega_{7,}\right) \cdot \omega_{t, v}{ }^{\prime}+M a_{t:} * v_{v}{ }^{r}
\end{aligned}
$$

For generalized rate $\oint_{j}\left[\omega_{x} \omega_{y} \omega_{z}\right]^{T}$ the generalized dominant force is $F_{\omega}$, the generalized inertial force is $F_{\omega}^{*}$.

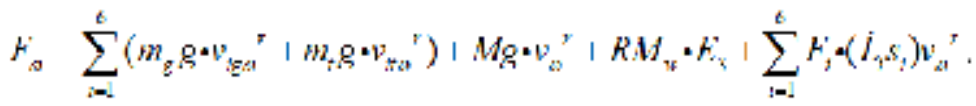

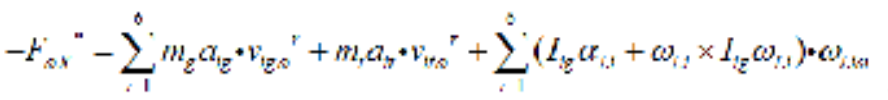

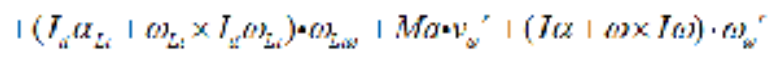

Where $F_{i}(i=1,2, \cdots \cdots, 6)$ is the driving force of each cylinder, $F_{w}$ is the external force acting on the center point $\mathrm{c}$ of the lower platform, and $M_{w}$ is the external moment acting on the lower platform.

The Kane kinetic equation is derived from the Dahl-Lagrangian principle:

$$
F_{j}+F_{j}^{*}=0 \text {. }
$$

According to (18) (22) to solve the driving force of each cylinder is $F_{i}(i=1,2, \cdots \cdots, 6)$.

\section{The Matlab and Adams co-simulation verification.}

The establishment of three-dimensional solid model. Solidworks is used to establish three-dimensional solid model of every part of the parallel wheeled robot. The assembly is completed and save the file as the Parasolid type for the next step import.

Co - simulation of Adams and Matlab / Simulink. Due to the complex motion control of parallel foot-wheeled robots, Adams can not meet the control requirements, So through the interface module Adams/ Controls of Adams and Matlab generate adams_sub file to achieve the data transfer between the two. In Matlab / Simulink to complete the establishment of joint simulation control system, so as to realize the control of the parallel foot-wheeled robot.

1) Perfect the model in Adams and generate the data transfer file with Matlab

Import the 3D solid model into Adams using the Parasolid file. Then, according to the actual motion relationship ,components are added with constraint, such as fixed Joints, the cylinder pair, the Hooker pair and so on in Adams. Model entity diagram shown in Figure 2.

Add the contact force between the wheel and the ground, add the drive to the cylinder pair and set the drive function to variable. The drive variables of the cylinder pair are set as input variables via the mechanical system derivation function of the Adams / Controls module. The output variable is set to contact force, the target function selected as Matlab, and then complete the data transfer between Matlab and file generation. 


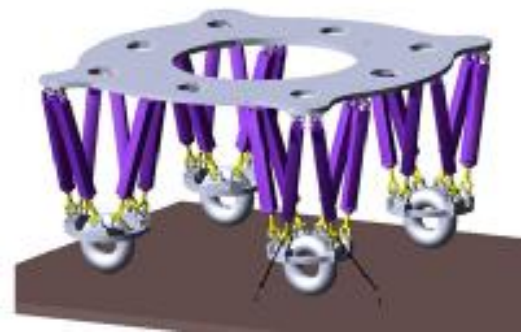

Fig.3 robot entity model Fig.

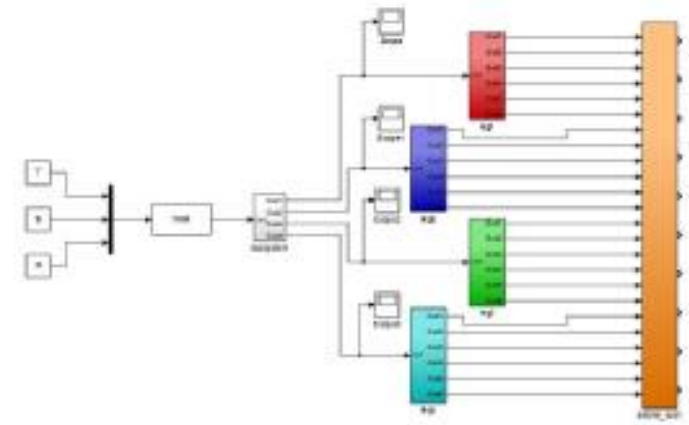

Fig.4 The control system of combined simulation

2) Build a joint simulation system

In Matlab, run the Adams generated $m$ file in the previous step, input the interface command Adams_sys, copy the generated subsystem module Adams_sub into Simulink and set up the joint simulation control system as shown in Fig.3

The system is an open-loop position control system, where $\mathrm{T}$ is the movement period of parallel foot-wheeled robot walking; $\mathrm{S}$ is the step length; $\mathrm{H}$ is the step height; Walk is the movement gait; The inputs from leg1 to leg4 are the movements of each leg in the gait, and the output is the displacement of each cylinder of the corresponding leg, which is the input to the model in Adams. Finally, by controlling the robot Walking cycle, step length, step height and movement gait to control the movement of each electric cylinder to complete the robot walking.

3) Co-simulation

Run the Co-simulation control system in Matlab / Simulink. After the simulation, the result file of res format will be imported into Adams, and the simulation result can be viewed by the post-processing module.

Results analysis. To the right movement, for example, the right leg of the robot for the first leg, the 1,2 cylinder position shown in Figure 2, the other cylinder in accordance with the clockwise sequence number. The results obtained from the kinetic equation are compared with those obtained by the co-simulation, as shown in Fig.5.
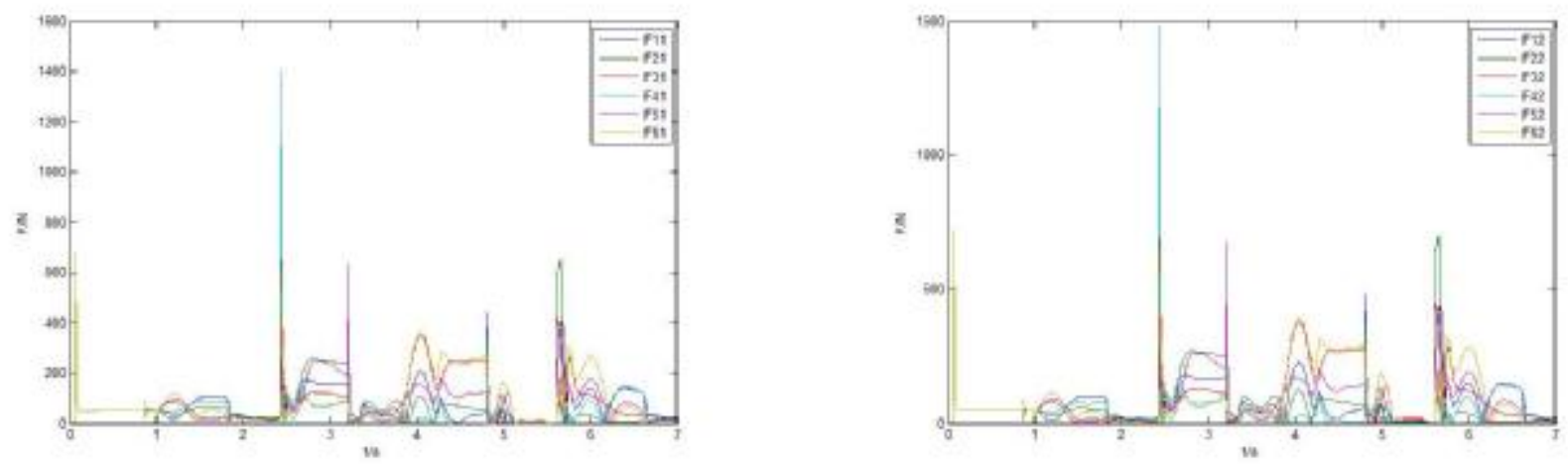

Fig.5 Comparison of results

Where F11 represents the driving force provided by the No.1 cylinder calculated by the kinetic equation,F12 represents the driving force provided by the No.1 cylinder obtained by the co-simulation method; The symbols in the other diagrams are the same. Because the theoretical analysis is in the ideal condition, there is a certain error with the simulation results. The difference between the two methods is small and the error is less than $7 \%$, which verifies the correctness of the dynamic model.

As shown in Fig. 5, at 2.5 seconds or so, the driving forces provided by the respective cylinders suddenly increase at the same time, which is caused by step 1 of the leg.

In the course of the whole movement, No. 3,4 cylinder of the maximum driving force was significantly greater than the other four cylinders, so in this gait on the 1 st leg,

As long as to ensure that the driving force of No. 3,4 cylinder is less than the maximum driving force providing by the cylinder itself the robot can move normally. 
On the one hand, you can adjust the robot gait method to reduce the maximum force between the various cylinder difference, so as to avoid a cylinder force is too large cause paralysis of the robot.

\section{Conclusions}

In this paper, two methods are used to analyze the dynamics of the robot, one is to establish kinetic equations using the kane method, the driving force of each cylinder during the motion of the robot is obtained, and the correctness of the established kinetic model is verified by the comparison of the results. The establishment of the kinetic model will provide the theoretical basis for the subsequent kinetics study. The simulation model can get the simulation data quickly and intuitively after the model is completed. It can improve the working efficiency and optimize the control system and structure size of the robot.

\section{References}

[1] Liming Peng. Mechanism Analysis of Virtual Axes Machine Tools Based on Virtual Prototyping Technology [D]. Zhejiang University, 2004.(In Chinese)

[2] Mengjie Jiang.A design for A 6-DOF Stewart platform and the research of the Application in the Optical testing[D]. Chinese Academy of Sciences(Changchun Institute of Optics, Fine Mechanics and Physics), 2012. (In Chinese)

[3] Xianrong Deng.Discussion about the Stewart Platform Mechanism for the Application in the Field of Radar[J].Electro-Mechanical Engineering, 2009, 25(2):41-43. (In Chinese)

[4] Lu Y, Li X P. Dynamics analysis for a novel 6-DoF parallel manipulator I with three planar limbs[J]. Advanced Robotics, 2014, 28(16):1121-1132.

[5] Wang S C, Hikita H, Kubo H, et al. Kinematics and dynamics of a 6 degree-of-freedom fully parallel manipulator with elastic joints[J]. Mechanism \& Machine Theory, 2003, 38(5):439-461.

[6] Li Y, Xu Q. Kinematics And Inverse Dynamics Analysis For A General 3-Prs Spatial Parallel Mechanism[J]. Robotica, 2005, 23(2):219-229.

[7] Ahmadi M, Dehghani M, Eghtesad M, et al. Inverse Dynamics of Hexa Parallel Robot Using Lagrangian Dynamics Formulation[C]// Automation Congress, 2008. Wac. 2008:1-6.

[8] Xiulong Chen, Dong Fangqi, Wang qing.Dynamic equation of 4-UPS-UPU parallel mechanism based on Newton-Euler approach[J]. Optics and Precision Engineering, 2015, 23(11): 3129-3137. (In Chinese)

[9] Cheng G, Shan X. Dynamics analysis of a parallel hip joint simulator with four degree of freedoms (3R1T)[J]. Nonlinear Dynamics, 2012, 70(4):2475-2486.

[10] Minjie Liu, Tian Yongtao, Li Congxin.Kane method for Substructure of the dynamics of Parallel Manipulators[J]. Journal of Shanghai Jiaotong University | J Shanghai ,2001, 35(7):1032-1035. (In Chinese)

[11] Shu Su.Kane method and its characteristics[J]. Journal of Machine Design, 1993(4):4-6. (In Chinese) 